RADIO SCIENCE Journal of Research NBS/USNC-URSI

Vol. 69D, No. 2, February 1965

\title{
Preface to "Waves in Plasma" Papers
}

The first thirteen papers of this issue deal with the propagation of electromagnetic waves in ionized media. The importance of this subject has increased significantly in recent years. With the advent of manned space vehicles and the need for communications during "reentry", the classical plasma sheath has ceased to be a laboratory curiosity. The alleviation of the "black-out" effects associated with the shock induced layer of ionized gas which surrounds a reentry vehicle is currently a pressing problem. Some of the papers in this issue deal directly with this topic. The first paper, by Dr. Bachynski of the RCA Research Laboratories in Montreal, serves as an excellent introduction to this topic.

The radiation characteristics of satellite-borne antennas which are immersed in ionized media (or plasma) such as the ionsphere are a related subject which recently has received a great deal of attention. In the paper by Professor Felsen, it is shown that a great deal of insight into this problem is obtained by studying the refractive index diagrams which relate the propagation vector for the homogeneous plasma with the oreintation of earth's magnetic field.

In most realistic situations, the plasma medium is not unbounded and homogeneous. Some very interesting effects are a result of the surface waves which may exist at the interface between the metallic surface of the vehicle and the plasma. A problem of this type is treated in the paper by Drs. Adachi and Mushiake of Tohoku University in Japan. By using a Fourier transform method, they find that surface waves will be excited by a magnetic line source on a plasma-coated conducting plane. Furthermore, it is shown that the characteristics of this wave may be controlled by the strength of a superimposed d-c magnetic field.

An important physical characteristic of a plasma layer is the profile of the electron density variation in the transverse direction. Recently, techniques have been developed to deduce this profile from data on the refraction of a microwave beam. In a short but important paper, Professor Cullen of Sheffield University discussed the validity of ray theory for this application.

When an antenna is immersed in the plasma, an important property is the driving point impedance. Even for antennas located in free space, this can be a difficult problem to treat theoretically. Many additional complications occur when dealing with inhomogeneous plasma as may be seen in the paper by Dr. Galejs of Sylvania Electric Products. Despite the complexity of the problem, he obtains specific results for the self and mutual admittance of waveguide-backed slots which radiate into a stratified plasma layer. 
In the classical theory of treating wave propagation in a simple plasma, it is assumed that collisions may be accounted for by assuming that the electron experiences a retarding force proportional to its velocity. This leads to the Appleton-Hartree formula for the two refractive indices of the ionized medium where the collision frequency is a constant. However, there is a growing body of experimental evidence which indicates that the collision frequency is approximately proportional to the square of the electron velocity. In a most enlightening paper, Dr. Budden of Cambridge University discusses the modifications required in order to generalize the theory. In the following paper, Dr. Mentzoni of Sylvania Electric Products uses microwave methods to determine the electron collision frequency in an oxygentype plasma. His results were consistent with the dependence according to the square of the electron velocity.

Now that radio signals are received on the ground from satellites, it is realized that the ionospheric plasma has numerous irregularities and perturbations. In a useful summary paper, Dr. Heisler of the University of Sydney in Australia gives an account of various experimental procedures for observing perturbations in ionospheric plasma. As he points out, a significant feature is the discrepancy between speeds and directions of perturbations measured by different observational techniques.

Some information about bounded plasma bodies (or idealized perturbations) can be obtained by measuring the radar cross section as a function of the scattering angle. Fundamental to a study is the influence of the parameters of the plasma on the strength of the scattered wave. By using a modified form of geometrical optics, Drs. Lee, Peters, and Walter of Ohio State University show how to calculate the radar cross section of an infinitely long cylinder of plasma which has a superimposed d-c magnetic field. They demonstrate the validity of their method by comparing their results with those calculated by using the known exact solution for the problem. In the following paper, Dr. Peters uses a similar approximate method to calculate the cross section of a spherical plasma body.

In most of the literature on "waves in plasma," including the above ten papers, the plasma is regarded as "cold." When a finite temperature plasma is considered, it is really necessary to consider the effect of finite compressibility. This means, in effect, that a source placed in a warm plasma will excite both electroacoustic- and electromagnetic-type waves. Then, in the limiting case of zero temperature, the electroacoustic wave ceases to propagate and the plasma becomes "cold." This subject, which has received considerable attention in the past year, is discussed in an interesting paper by Dr. Chen of Michigan State University. In a companion paper, he also considers the effect of finite compressibility on the radar return from orbiting bodies immersed in the ionospheric plasma. Dr. Chen suggests that the large outbursts of the observed reflected HF signals from satellites may be attributed to the hitherto neglected electroacoustic wave. It is clearly evident that this subject warrants further study.

The final paper in the issue, contributed by the editor, also deals with electroacoustic and electromagnetic waves in a compressible plasma. It is shown that a cylindrical inhomogeneity in a warm plasma will serve as a mechanism for conversion of electromagnetic modes to electroacoustic modes and vice versa.

It is planned that the following issue of Radio Science will contain additional papers on "waves in plasma" in addition to the regular contributions. 